\title{
Efficient stray-light suppression for resonance fluorescence in quantum dot-micropillars using self-aligned metal apertures
}

\author{
Caspar Hopfmann ${ }^{1}$, Anna Musial ${ }^{*, 2}$, Sebastian Maier ${ }^{3}$, Monika Emmerling ${ }^{3}$, \\ Christian Schneider ${ }^{3}$, Sven Höfling ${ }^{3,4}$, Martin Kamp ${ }^{3}$, and Stephan Reitzenstein ${ }^{1}$ \\ ${ }^{1}$ Institute of Solid State Physics, Technische Universität Berlin, D-10623 Berlin, \\ Germany \\ ${ }^{2}$ Laboratory for Optical Spectroscopy of Nanostructures, Division of Experimental \\ Physics, Faculty of Fundamental Problems of Technology, Wrocław University of \\ Science and Technology, 50-370 Wrocław, Poland \\ ${ }^{3}$ Technische Physik, Physikalisches Institut and Wilhelm-Conrad-Röntgen-Resarch \\ Center for Complex Material Systems, Universität Würzburg, D-97074 Würzburg, \\ Germany \\ ${ }^{4}$ SUPA, School of Physics and Astronomy, University of St Andrews, KY16 9SS St. \\ Andrews, United Kingdom \\ *E-mail: anna.musial@physik.tu-berlin.de
}

\begin{abstract}
Within this work we propose and demonstrate a technological approach to efficiently suppress excitation laser stray-light in resonance fluorescence experiments on quantum dot-micropillars. To ensure efficient stray-light suppression, their fabrication process includes a planarization step and the subsequent covering with a titanium mask to fabricate self-aligned apertures at the micropillar positions. These apertures aim at limiting laser straylight in side-excitation vertical-detection configuration, while enabling detection of the optical signal through the top facet of the micropillars. Beneficial effects of these apertures are proven and quantitatively evaluated within a statistical study in which we determine and compare the stray-light suppression of 48 micropillars with and without metal apertures. Actual resonance fluorescence experiments on single quantum dots coupled to the cavity mode prove the relevance of the proposed approach and demonstrate that it will foster further studies on cavity quantum electrodynamics phenomena under coherent optical excitation.
\end{abstract}

PACS: 78.67.Hc (optical properties of QDs), 42.50.Ct (light interaction with matter), 87.67.-n (optical properties of low-dimensional structures), 42.50.Pq (cavity quantum electrodynamics), 78.55.Cr (photoluminescence of III-V semiconductors)

\section{Introduction}

In the active field of cavity quantum electrodynamics (cQED), quantum dot (QD) - microcavities are prominent candidates for the investigation of light-matter interaction effects [1-4] in both the weak and the strong coupling regime [5,6]. As such they are of high interest from a fundamental point of view and are also very attractive candidates for the realization of, e.g., single photon sources [7], as building blocks for long-range quantum networks, e.g., quantum repeaters [8] and optical implementations of quantum computing [9]. One prominent example is the micropillar geometry, which has attracted significant interest in recent years [10]. They allowed, among others, for the first observation of strong 
single exciton-single photon coupling [5], for realization of efficient optically- and electrically-driven single photon sources [11], and for on-chip integrated quantum optical circuits [12]. They have also proven to be beneficial to study nonlinear and laser dynamics in the quantum regime [13]. In most publications in this context, non-resonant excitation schemes were used to investigate cQED effects of interest. Recently, also resonance fluorescence (RF) has started to be exploited in this regard with a focus on, e.g., the study of off-resonant QD-cavity mode coupling [16], the generation of highly indistinguishable single photons [15, 16], as well as laser field-QD [17] coupling. RF is of particular interest since it allows for direct coherent access to all QD states without the detour of relaxation processes inducing additional time jitter and dephasing $[1,18,19]$, the suppression of which is very beneficial for, e.g., enhancing the indistinguishability of emitted photons [19, 20]. Resonant excitation is also crucial for coherent, all-optical QD state control [21] as well as for coherent preparation of selected states which are inaccessible in non-resonant photoluminescence experiments, such as twophoton excitation of QD biexciton [22].

The inherent and the biggest challenge of RF experiments is the efficient suppression of the excitation stray-light, because of the wavelength degeneracy of the pump and emission signal, where the excitation density is typically at least six orders of magnitude larger than that of the fluorescence. Two viable approaches to overcome these challenges have been developed in recent years: polarization filtering utilizing linear cross-polarization of excitation and detected RF signal [15, 16, 19, 23, 24] and spatial separation of the excitation and detection path [14, 17, 25]. Both methods have particular limitations making them not fully suitable to comprehensively study QD-microcavity systems. Polarization filtering is experimentally readily realizable and has been proven very efficient in the case of flat unstructured surfaces without cavities [23], but in the case of QDs in structured nano- or microphotonic devices, such as small diameter micropillar cavities, this scheme is less effective due to non-polarization preserving scattering processes on structured material as well as structural imperfections. The concept of spatial separation of the excitation and detection beam path, on the other hand, cannot be applied to all structures (depending on the details of their geometry) and requires a more complex experimental setup since the excitation and detection beam paths need to be aligned with respect to each other as well as to the sample on a sub-micrometer scale. The spatial separation is of particular advantage in case of QDs embedded in micropillar cavities, because it allows for flexible and efficient excitation regardless of the spectral properties of the cavity stopband which modulates and reduces the excitation efficiency in axial direction. Moreover, if used solely (without additional cross-polarization filtering), lateral excitation promises to double the detection efficiency in axial direction, which is of particular importance for the realization of bright single photon sources. To maximize the RF stray-light suppression, additional spatial filtering of the detection beam path using a micrometer sized pinhole can be employed. It is noteworthy that both spatial as well as polarization filtering decreases in efficiency if the investigated microstructures are on the same spatial scale as the $\mathrm{RF}$ wavelength, since, e.g., non-polarization preserving scattering processes cannot be filtered entirely. To overcome this spectroscopic limitations, it is very desirable to introduce additional structural optimizations (sample design) for advanced RF filtering which would enable to study cQED phenomena in QD-microcavities under resonant excitation in the low diameter limit where on one hand light-matter interaction is most pronounced, but on the other the contribution of the stray-light to the signal detected in the vertical direction is the largest. This is of particular importance when focusing on strong coupling effects which require particularly low mode volumes and are most pronounced in micropillars with diameters below $2 \mu \mathrm{m}$.

In this study we propose and evaluate an advanced structural stray-light suppression element, i.e. selfaligned metal microapertures above the top facet of QD-micropillars. The aim of this element is to limit the signal detected in the vertical direction to the radiation through the top facet of the micropillar by blocking the stray-light of the micropillar sidewalls in analogy to a pinhole in the optical setup, but much more precise, perfectly self-aligned to the micropillar top facet and not affected by the aberrations and not perfect alignment of the optical imaging system. We combine the metal apertures with the methods of excitation stray-light suppression described above and present RF experiments on single QD excitons coupled to the fundamental mode (FM) of a micropillar cavity. This advanced sample design will foster comprehensive investigations on resonantly-driven cQED effects in micropillar cavities in particular in the strong coupling regime, since the metal mask reduces the stray- 
light of micropillar sidewalls significantly with particular benefit in the low micropillar diameter range. The reduction of the stray-light is studied experimentally and the strength of this effect is evaluated quantitatively for micropillars with different diameters with and without metal mask.
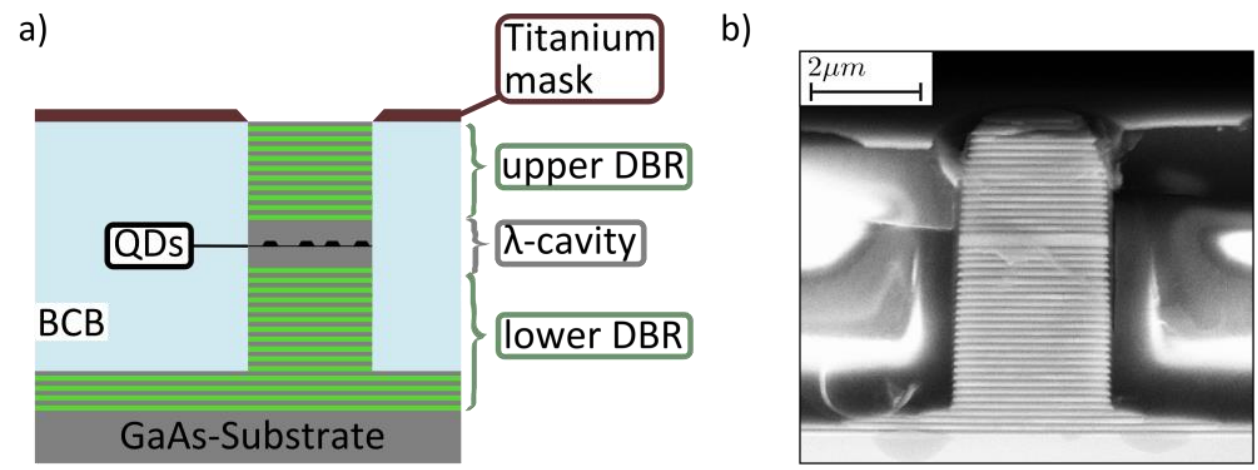

c)

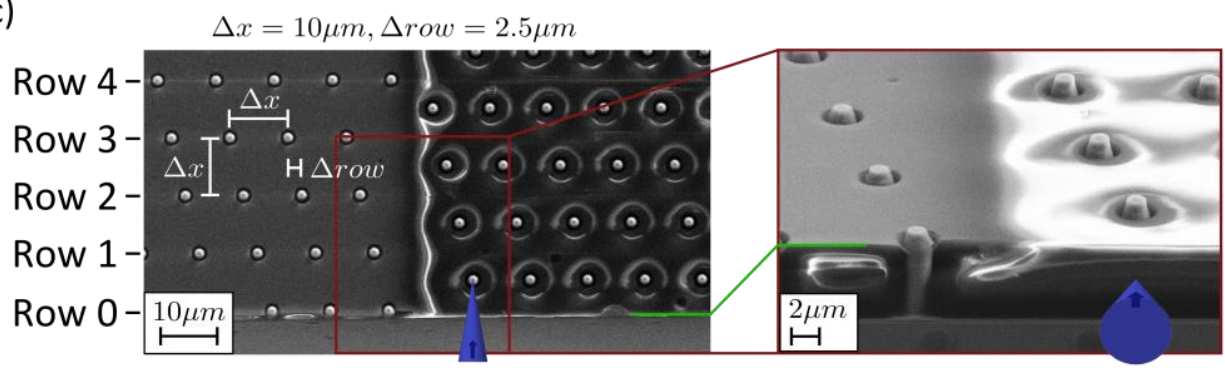

Figure 1. (a) Schematic of a QD-micropillar cavity with titanium mask containing self-aligned microapertures. (b) Scanning electron microscope (SEM) picture of a micropillar cross-section of nominally $2.0 \mu \mathrm{m}$ diameter. (c) Left: Top-down SEM picture of $1.5 \mu \mathrm{m}$ diameter micropillars with titanium mask on the left half and without mask on the right half. Due to the dielectric nature of the BCB the SEM picture shows a characteristic charging artifact (rings around micropillars). The sample edge and the direction of side-excitation are indicated in green and blue, respectively. The micropillars are arranged with an offset of $2.5 \mu \mathrm{m}$ per row $(\Delta \mathrm{row})$ in vertical direction fostering accessibility from the edge using side excitation. Right: Enlarged SEM picture recorded using a $60^{\circ}$ tilt.

\section{Experimental setup and sample preparation}

In our experiments we employ QD-micropillar structures realized by high-resolution electron-beam lithography and reactive ion etching from a planar epitaxially grown microcavity wafer. The wafer substrate is GaAs on which consecutively a bottom distributed Bragg reflector - DBR (using alternating AlAs and GaAs layers of nominal thicknesses of 83 and $69 \mathrm{~nm}$, respectively) with 25 mirror pairs, a central cavity of $277 \mathrm{~nm}$ nominal height and a top DBR with 15 mirror pairs is grown using molecular beam epitaxy (MBE). A layer of partially capped $\operatorname{In}(\mathrm{Ga}) \mathrm{As} / \mathrm{GaAs}$ QDs of low surface density $\left(5 \times 10^{9} \mathrm{~cm}^{-2}\right)$ is embedded centrally into the one- $\lambda$ thick GaAs cavity layer, enabling the investigation of single QD excitonic complexes and their interaction with micropillar cavity modes. Micropillars with diameters between 1.5 and $3.0 \mu \mathrm{m}$ are realized by reactive ion etching and feature close to perfect vertical sidewalls. Subsequently, benzocyclobutene (BCB) is used to planarize the sample. Further details of the fabrication process and underlying layer design of similar micropillar structures are outlined in references 11, 26 and 27. The next step - namely fabrication of the microapertures within the lift-off process exactly at the positions of the micropillars - is crucial for the proposed technological approach as it ensures that the upper facets of the micropillars are free of any absorbing material. The self-alignment to the micropillar top facets is achieved by using the $750 \mathrm{~nm}$ thick barium fluoride-chromium $\left(\mathrm{BaF}_{2} / \mathrm{Cr}\right)$ mask twice during the whole process: first as the mask for dry etching of the micropillars and subsequently (using the remaining ca. $500 \mathrm{~nm}$ of $\mathrm{BaF}_{2} / \mathrm{Cr}$ ) as a liftoff mask for the $250 \mathrm{~nm}$ thick titanium film. This film ensures efficient suppression of laser stray light in normal direction with suppression ratio close to $10^{6}$ typically required for single QD RF experiments. In this way, half of the micropillars (of each size) of the present sample are provided with 
metal apertures, while the others act as reference structures to evaluate the impact of the local apertures. A schematic and microscopic cross-section image of exemplary micropillar structures is depicted in figure 1(a) and (b), respectively. The micropillars with DBR mirror design as described above feature moderate cavity quality (Q-) factors of about 2500 for $2.0 \mu \mathrm{m}$ diameter micropillars and are optimized for efficient outcoupling of QD emission energetically close to the cavity resonances.

After processing, the sample is cleaved to enable optical access to the embedded QDs in the lateral direction, i.e. side-excitation as used in this study. At the same time it opens up a possibility to collect the signal in the lateral direction, e.g., to monitor the direct QD spontaneous emission into free space or loss channels of the micropillar cavity [28]. The micropillars are structured into the wafer material using a rhombic pattern with an offset ( $\Delta$ row) of $2.5 \mu \mathrm{m}$ for each row of micropillars counting from the cleaved edge. In figure 1(c) the layout of the micropillar structures close to the cleaved edge is shown. The intended role of this rhombic pattern of the micropillar array is to increase the number of micropillar structures available for side-excitation, since it prevents shadowing of micropillars in the first four rows from micropillars closer to the edge and potentially enhances stray-light suppression by spatially separating light scattered by the cleaved edge from the micropillar top facets.

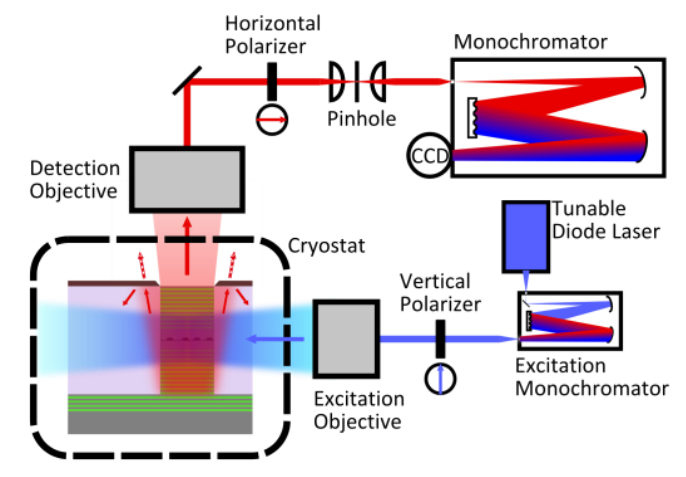

Figure 2. Schematic of the side excitation - vertical detection experimental setup, the mechanism of intended advanced resonance fluorescence filtering using metal microapertures is indicated (within dashed border). Elements are not to scale.

For our spectroscopic studies the micropillar sample is mounted inside a helium flow cryostat and kept at a constant temperature of $15 \mathrm{~K}$. We employed an experimental setup (figure 2) in which we excite QDs embedded in micropillars via side-excitation (blue), and detect emission through the top facet using a second independent beam path (red) $[14,28]$. In the side-excitation beam path we employ a long working distance microscope objective with a numerical aperture (NA) of 0.4 and magnification of 20 times and in the detection beam path an objective with NA $=0.65$ and 50 times magnification. In an ideal case, i.e. in the absence of non-direction preserving scattering processes, this $90^{\circ}$ excitationdetection scheme should enable perfect suppression of the excitation laser, which is especially relevant when performing RF experiments. In reality however, diffraction and scattering processes, which are neither direction nor polarization preserving, take place at the interfaces of the micropillars, limiting the suppression of the excitation laser by the $90^{\circ}$ excitation-detection scheme to commonly six orders of magnitude. To reduce the related stray-light we employ a pinhole of $20 \mu \mathrm{m}$ diameter in the detection path and we polarize the excitation beam linearly and select emission polarized in the orthogonal plane in the detection path to further increase the excitation stray-light suppression. The detected light is analyzed in a high-resolution spectrometer equipped with a liquid nitrogen-cooled low-noise charge coupled device (CCD) detector, featuring a spectral resolution of down to $25 \mu \mathrm{eV}$. Great care is taken to make sure that the excitation and detection objective foci coincide on the same point on the sample, to optimize this excitation-detection scheme. For RF experiments a mode-hop free tunable diode laser featuring a full width at half maximum (FWHM) linewidth below $100 \mathrm{kHz}$ $(0.41 \mathrm{neV})$ is employed. For non-resonant excitation experiments a stabilized frequency-doubled neodymium-doped yttrium aluminium garnet laser at $532 \mathrm{~nm}$ emission wavelength is used. A schematic picture of our experimental setup is shown in figure 2. Note that all RF experiments shown in this study are performed employing very weak background illumination of $<<1 \mathrm{nW}$ (not shown in 
figure 2 for simplicity) used to fill charge traps in the vicinity of the QDs and therefore reduce QD dephasing and spectral jitter [29].

\section{Experimental observations}

At first, basic optical properties of the QD-micropillars are described with special focus on possible modifications of their spectra due to the metal layer. It is important to note that the additional technological steps such as the planarization and patterning of the metal mask do not deteriorate the optical properties of the structures in terms of the Q-factor necessary for the observation of cQED effects $[3,5,28]$. It is also crucial for further comparison of the excitation stray-light suppression that the optical properties of the micropillars of the same diameter with and without metal do not differ substantially. To confirm this, emission spectra from micropillars of different diameters with and without metal mask have been first studied under above bandgap excitation (figure 3). Exemplary micro-photoluminescence $(\mu \mathrm{PL})$ spectra recorded at QD saturation excitation power (in the range of $50 \mu \mathrm{W}$ measured outside the cryostat) of two QD-micropillars with $2 \mu \mathrm{m}$ nominal diameter with (red) and without (black) metal cover respectively, are presented for comparison. Micropillar cavities with embedded QDs show characteristic mode spectra featuring a low energy cut-off given by the fundamental mode (FM - grey area in figure 3) $\mathrm{HE}_{11}$ which is twofold energetically degenerate in systems with ideal in-plane rotational symmetry. Higher order radial modes emerge at higher energies in a pattern characteristic for the micropillar diameter. Note that the energetic position of the fundamental mode differs significantly by $5.1 \mathrm{meV}$ between the two presented micropillars, even though the nominal dimensions are the same. This energy difference is related to a radial inhomogeneity of the DBR-mirror layer thicknesses across the wafer induced in the epitaxial growth process - one has to keep in mind that the micropillars with equal diameters are spatially separated (even up to few $\mathrm{mm}$ ) due to the fact that half of the sample is covered with metal and the micropillar sizes are spatially grouped together. Beyond that, comparison between previous studies on various QD-micropillar samples without metal apertures and the investigated sample (not shown here) indicate that the position of the FM is not influenced by the presence of metal masks. Similar systematic dependences of the cavity resonance energy as a function of the spatial position with respect to the wafer center (for a given pillar diameter) were observed in all cases. This results in a shift of the cavity resonance with respect to the maximum of the QD ensemble emission, which also influences the emission intensity of the micropillar. As a result, the inhomogeneity in the layer thicknesses within the sample were identified as the main mechanism differentiating the resonance frequency in the case of pillars with equal diameters independent of the presence of the metal layer. Moreover, no statistically significant differences in brightness and Q-factors between micropillars of the same diameters from the two parts of the sample (with and without metal mask) have been observed, which proves the preservation of high structural quality in the extended technological process. We would like to mention that on average we do not observe indications of strain-related effects, i.e. possible spectral shifts of the cavity mode or the QDs, due to the metal mask. For both types of micropillars, with and without metal microapertures, the Q-factor decreases with reduced micropillar diameter as surface scattering of the FM and the associated optical losses, increase [26, 28]. The active medium of the investigated structures consists of an ensemble of QDs with ground state energies statistically distributed according to the QD material composition and spatial dimensions [30]. To facilitate single QD experiments, the maximum of this distribution is designed to be blueshifted with respect to the FM energy. Emission of single QD excitonic complexes (figure 3) with energies close to the modes of the micropillar resonator show higher intensity because of a cavity-enhanced outcoupling of emission [31]. At higher energies, higher order modes and wetting layer emission (green) can be observed in the spectrum. Moreover, vertical emission of spectrally off-resonant QD lines is efficiently suppressed for micropillars with metal apertures. 


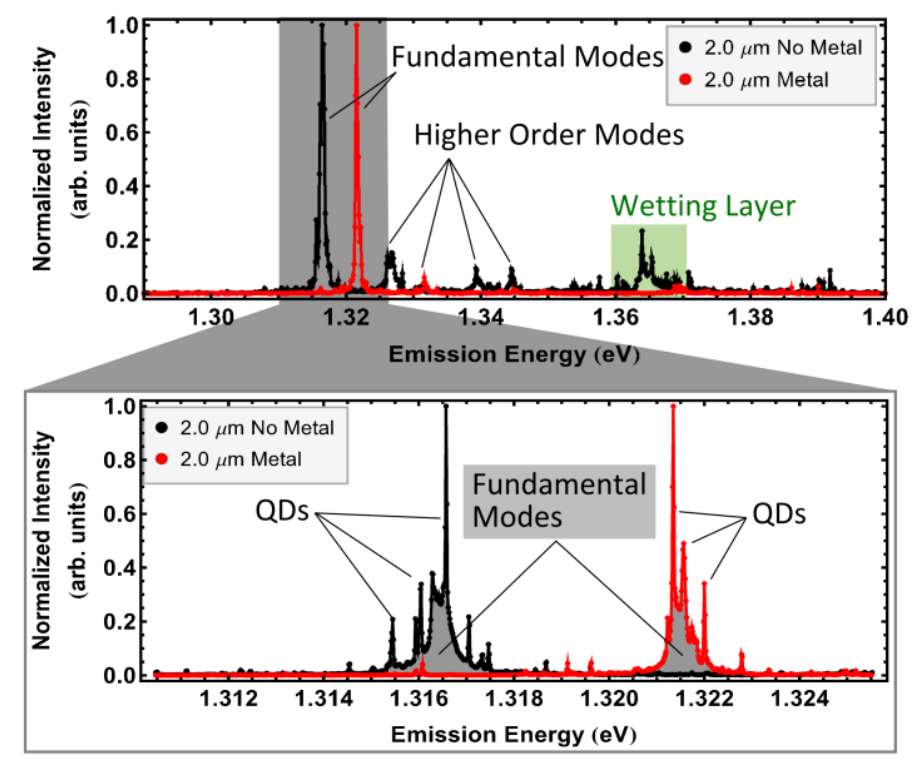

Figure 3. Top: Overview photoluminescence (PL) spectrum using above-band excitation at QD saturation of 41 and $55 \mu \mathrm{W}$ excitation power of two $2.0 \mu \mathrm{m}$ diameter micropillars with and without metal mask, respectively. Bottom: Close-up micro-PL spectrum of the fundamental modes (FMs). Individual cavity enhanced quantum dot (QD) emission lines are clearly visible near the FMs.

To verify whether the metal-masked micropillars feature enhanced suppression of laser excitation and to evaluate this effect quantitatively, the laser energy was varied in the range of $4 \mathrm{meV}$ across the energy of the FM and photon leakage from the cavity in the vertical direction was detected. Such resonant laser scans were taken for each available micropillar diameter of 1.5, 2.0, 2.5 and $3.0 \mu \mathrm{m}$ using side-excitation and axial detection for at least five micropillars of each diameter with and without metal mask. Constant laser power of $5 \mu \mathrm{W}$ far beyond single QD saturation $\left(\mathrm{P}_{\text {sat }} \approx 150 \mathrm{nW}\right.$ under resonant excitation) prevents single laser-QD resonances to impact the results significantly, thereby ensuring that the signal detected originates from scattered resonant laser light. Under these experimental conditions, the related RF signal will be negligible in comparison to the intensity of the scattered laser light. However, if a QD is in resonance with the cavity mode, the excitation will be transferred to the mode and as a result a resonance at the mode energy can be observed. Three exemplary scans are shown in figure 4(a): two for uncovered micropillars (black symbols) and one for a metal covered micropillar (red symbols) with nominal diameters of $3.0 \mu \mathrm{m}$. The enhancement of the signal coinciding with the FM energy for the two presented curves is attributed to cavity enhanced emission of resonant QD (figure 4(a) - full symbols). If no QDs are in resonance with the cavity mode no change of scattering light intensity is expected at FM energy (figure 4(a) - open symbols). For micropillars without metal mask, all light scattered in the vertical direction can in principle be detected (this includes scattering at micropillar sidewalls and other interfaces in the microstructure), but the metal layer should limit the detected signal to the scattered light through the facet of the top DBR (cf. figure 2). This reduction of scattered light detected in the vertical direction is clearly visible in figure 4(a) - the intensity of scattered light is about one order of magnitude lower if compared to micropillars without metal aperture. To enable a quantitative comparison of these curves regardless of QD-FM resonances the minimum value of each curve, which is related to the base level of the scattered laser light since it does not include any resonance effects, is taken. Logarithmic means of these minimum values for a given micropillar diameter and micropillar type (with and without metal) are plotted in figure 4(b) including indication of the statistical error (upper plot). The statistical deviations from the logarithmic mean value of the minimum values for RF laser emission energy scans are quite significant. The reason is that the scattering is very sensitive to any structural imperfection of the micropillar, e.g., at the sidewalls, but also at the interfaces of the DBR sections. Deviations from the mean value of micropillars with metal mask are generally larger than for micropillars without it, indicating that imperfections in the metal mask can lead to additional micropillar-to-micropillar variation. In the lower plot the scattered light suppression enhancement factor, defined as the ratio of mean values of micropillars without metal mask to micropillars with metal mask (corresponding to the 
increase in signal to background ratio between the two micropillar types) is presented as a function of the micropillar diameter. For all investigated diameters the micropillars with metal mask show significantly better stray-light suppression, reflected in lower values of the intensity of scattered laser light compared to micropillars without metal mask. The suppression enhancement factor is diameter dependent and lies in the range of 3 to 15 .

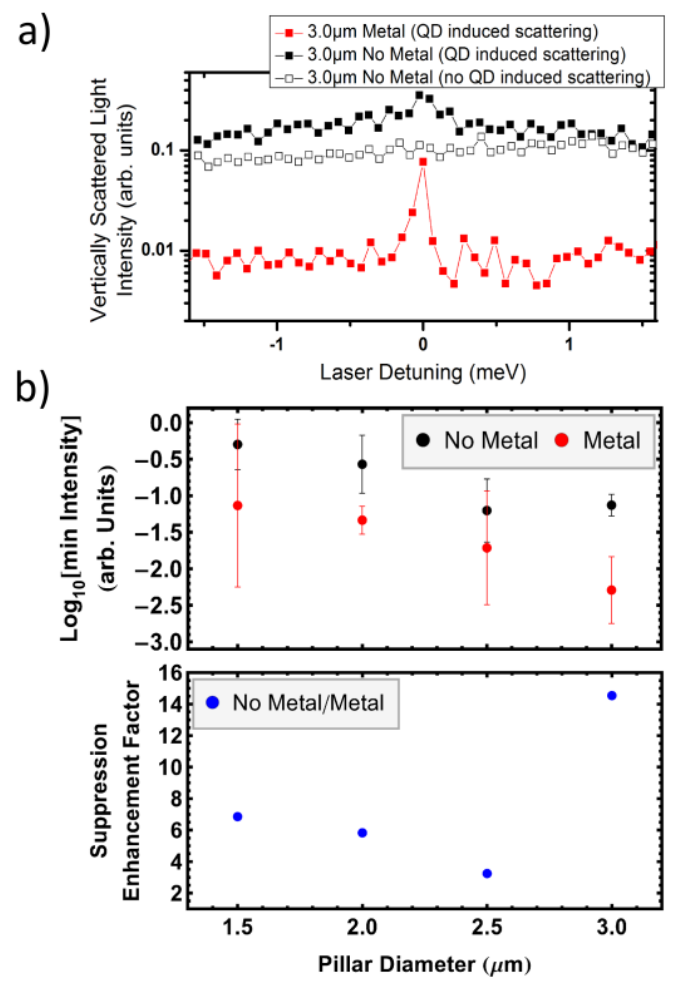

Figure 4. (a) Exemplary resonant laser scans across the FM energy (zero detuning) at $5 \mu \mathrm{W}$ excitation power for $3.0 \mu \mathrm{m}$ micropillars with (red) and without (black) metal cover with presence of additional quantum dot (QD)induced upward scattering (full symbols). (b) Top: Logarithmic mean values of the minimum of the intensity of light scattered in the vertical direction for micropillars with (red) and without (black) metal mask, respectively. The mean value and statistical error are calculated from multiple samples (minimum of 5 samples per diameter and type). Bottom: Stray-light suppression enhancement factor due to metal aperture for different micropillar diameters.

Micropillars with enhanced laser light suppression using metal masks are especially favorable for single QD RF experiments at low intensities. To illustrate that, an exemplary RF study on single QDs coupled to the FM of a micropillar cavity with a diameter of $2.0 \mu \mathrm{m}$ was performed. At first, RF from a single QD and its coupling to the cavity mode as well as cavity-mediated coupling to other QDs was demonstrated and additionally the influence of excitation power on the RF intensity as well as QD saturation behavior was investigated. RF signal from a single QD was obtained by tuning the laser emission energy across the transition energy of QD1 (the laser energy is indicated in the 2D map in figure 5 by a blue line) - cf. above band gap spectra (red) in figure 5 top right-hand corner - using side excitation at $60 \mathrm{nW}$ excitation power (well below QD saturation). The response of the system was evaluated in terms of intensities integrated over various spectral ranges depending on the spectral linewidth of the respective feature. The signal detected at the excitation laser energy (blue solid line in the 2D map) including QD-related RF and residual laser stray-light is cut out of the 2D-image for scaling reasons as it is much stronger than the resonances at the energy of the FM and another QD (marked as QD3 in the above bandgap spectra) spectrally overlapping with the FM. The color-coded rectangles shown in the $2 \mathrm{D}$ map indicate the spectral regions of integration. The respective dependencies of the integrated intensity vs. QD-laser detuning are presented in the graphs below for RF (blue), QD3 (purple) and FM (green). 


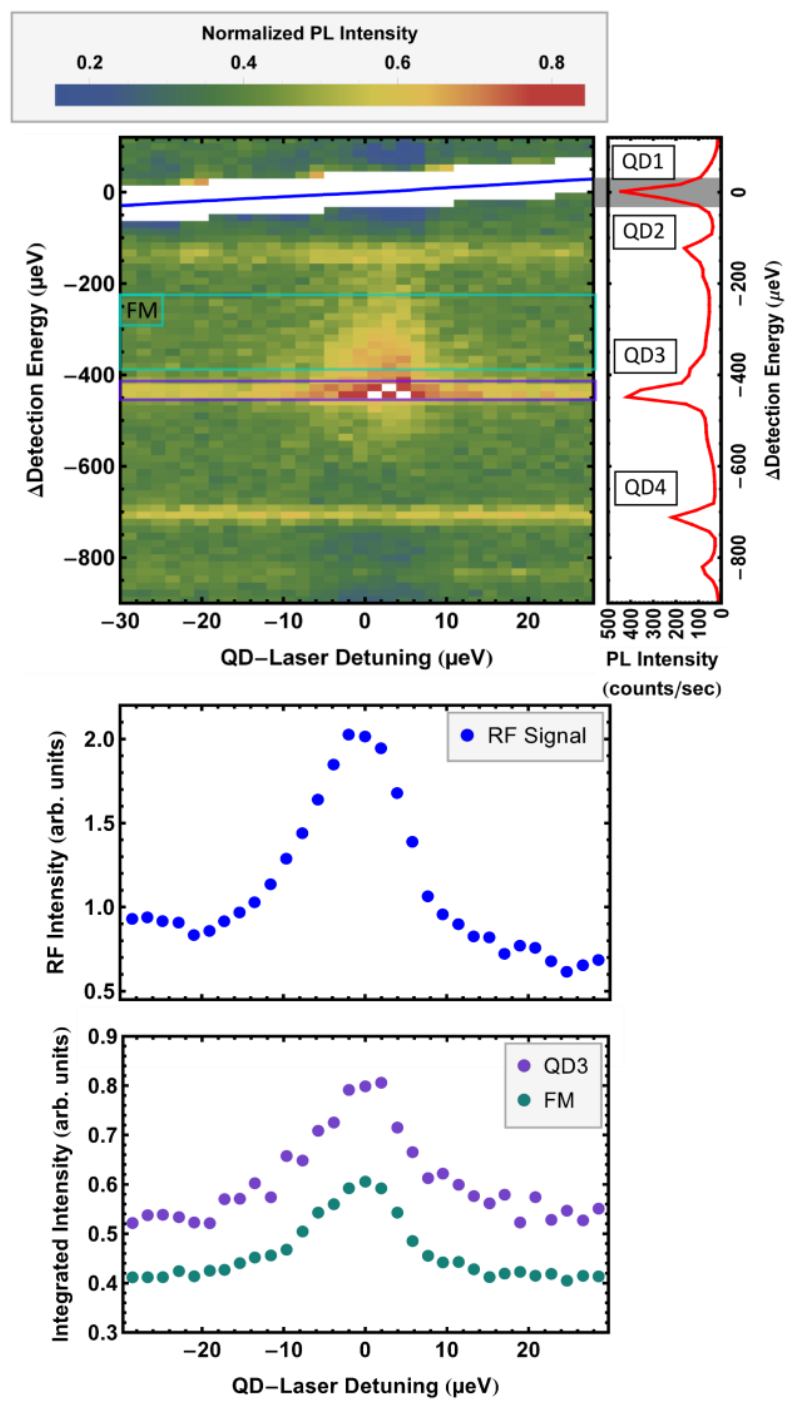

Figure 5. 2D luminescence map of a $2.0 \mu \mathrm{m}$ diameter micropillar with metal mask obtained by scanning a laser across a quantum dot's (QD1) s-shell transition using $60 \mathrm{nW}$ side-excitation. All energetic scales are given in relation to the QD1 transition energy. On the right to the 2D PL-intensity map, a low intensity above-band PLspectrum at $20 \mathrm{nW}$ excitation power is shown for comparison (red). Below the 2D-map, the integrated RF intensity - blue (which was cut out of the 2D-map for scaling reasons), as well as the integrated intensities at the energies of the QD3 transition (purple) and the fundamental mode (FM - green) as a function of detuning between QD1 resonance and laser are shown.

Both the RF signal as well as the mode response show clear resonances if the laser is resonant with the s-shell transition of QD1 with a width of the s-shell resonance of about $11 \mu \mathrm{eV}$. The resonance at the FM energy can be used as a monitor of the RF of excited QD [14] and indicates that off-resonant QDFM coupling is present. While the amplitude of the s-shell RF signal is slightly larger than 2, the increase in the signal of the mode is only by about a factor of 1.5 . The lower amplitude of the mode response indicates that the off-resonant QD-FM coupling is not solely and linearly dependent on the QD exciton occupation (comparing only the amplitudes, so the increase in the signal on resonance, and not the absolute signal level, accounts for the non-perfect < 1 efficiency of the excitation transfer). The off-resonant QD-FM coupling is likely provided by phonons as reported in [14, 32], since we do not expect energy transfer via continuum of wetting layer states under resonant excitation cf. references 33 and 34 . The presence of the resonance (very similar to that observed for FM) at the QD3 transition energy (figure 5 - bottom graph) can be explained by the QD3-FM coupling (QD3 is spectrally in resonance with FM). This implies that the s-shell resonance of QD1 is imprinted onto QD3 via consequent phonon coupling of QD1-FM and resonant cQED coupling of FM-QD3. The FM can therefore be used to prepare a resonant state of a QD through pumping another QD's s-shell at a different energy and utilizing a FM-mediated interaction between QDs coupled to common optical 
field. Other QDs - such as QD4 (out of resonance with the FM) - do not show resonance behavior, indicating that this effect is related to the resonant FM-QD coupling.
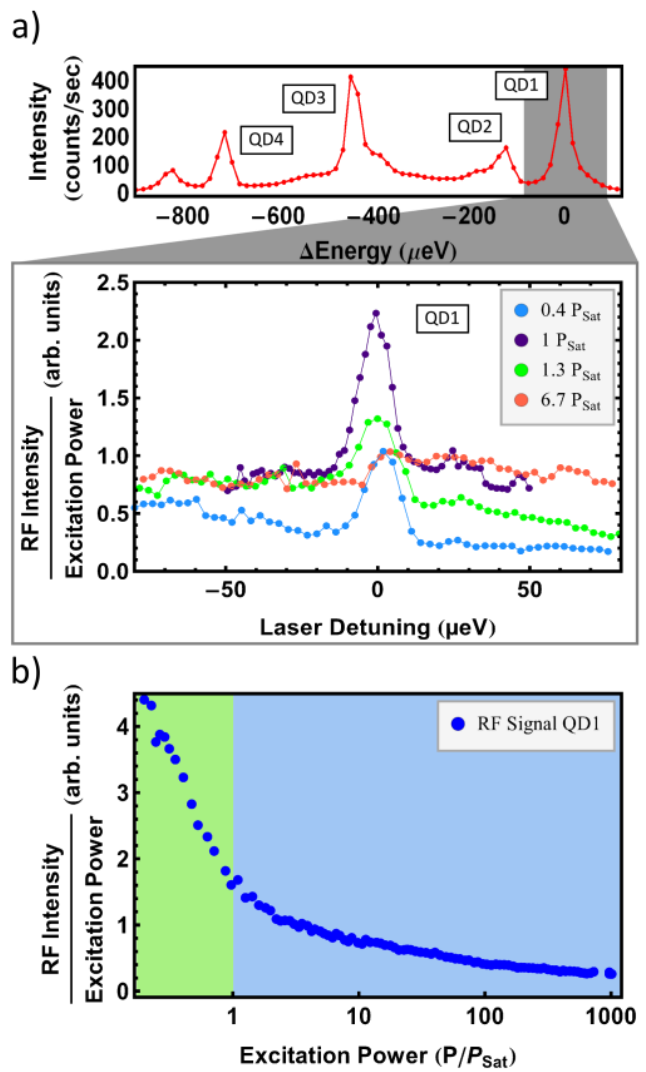

Figure 6. (a) Top: Photoluminescence (PL) spectrum under low power ( $20 \mathrm{nW}$ ) above band excitation. Bottom: Integrated resonance fluorescence $(\mathrm{RF})$ intensity signal normalized by excitation power for selected excitation intensities given with respect to the quantum dot $\mathrm{QD} 1$ saturation power $\left(\mathrm{P}_{\mathrm{sat}} \approx 150 \mathrm{nW}\right)$. All detunings are given with respect to the QD1 resonance energy. (b) Integrated RF intensity of QD1 normalized by excitation power as a function of excitation power relative to the QD1 saturation power. Excitation powers below and above QD1 saturation are indicated with green and blue regions, respectively.

The response of the QD s-shell resonance is dependent on excitation power, since the QD will be saturated and therefore becomes transparent above the saturation excitation power $\left(\mathrm{P}_{\text {Sat }}\right)$ determined to be $150 \mathrm{nW}$ for QD1. Figure 6 presents results obtained by excitation power dependent resonant laser scans across QD1 transition for powers below, equal to and above $\mathrm{P}_{\text {Sat }}$ (figure 6(a)). It is clearly visible that the power normalized s-shell resonance emission intensity of QD1 is strongest at saturation and decreases at excitation powers below and above $\mathrm{P}_{\text {Sat. }}$. At this excitation power the signal to background level is at least 2.6, implying that on resonance for every background photon $\geq 2.6$ photons are emitted/scattered by the QD through the micropillar top facet. No background subtraction was performed within the data evaluation process and therefore no additional sources of scattering of the side excitation laser are accounted for. Since additional sources - such as other QDs, micropillar modes or defects - are present and contribute to the background level, the RF-signal to background ratio of 2.6 represents the lower limit of the QD RF to laser stray-light photons. In figure 6(b) the power normalized RF signal intensity at zero QD1-laser detuning as a function of excitation power is depicted. In this plot a constant normalized RF intensity would indicate a linear response of QD1 induced resonant emission/scattering. A decreasing intensity curve would indicate a sub-linear QD1 response. The depicted normalized intensity curve shows a signal decrease with two different slopes which corresponds to a sub-linear QD response and therefore saturation behavior: At low intensities the response of QD1 is strongly sub-linear (strong decrease in normalized RF in green region in figure 
6(b)), whereas above the estimated QD1 saturation, the dependence becomes almost flat indicating nearly linear response of the QD (blue region in figure 6(c)). This behavior can be explained by the emission/scattering of the QD remaining constant above saturation and only the background emission increasing linearly due to increased excitation power.

These exemplary RF results prove the relevance of applied approach for study on small diameter (low mode volume) QD-microcavity systems and the advantage brought by our advanced sample design. This paves a way towards studying of the coherent QD-FM coupling regime under resonant excitation.

\section{Conclusions and outlook}

In this work we proposed and evaluated an advanced sample design for effective suppression of light scattered in the vertical direction in semiconductor microstructures. This approach relies on apertures in a titanium mask that are self-aligned to the micropillar positions and is very beneficial for RF study on patterned surfaces and microcavities, in particular application relevant QD-micropillars providing comprehensive insight into cQED phenomena under resonant excitation. The suppression of stray light was evaluated quantitatively by measuring the laser light scattered by QD-micropillar cavities with and without self-aligned metal microaperatures using a side-excitation vertical-detection scheme. The excitation laser suppression is increased by a factor which varies from 3 to 15 verified for various micropillar diameters $(1.5,2.0,2.5$ and $3.0 \mu \mathrm{m})$. Micropillars employing a self-aligned metal mask allow single QD-RF experiments in micropillars proving usefulness of the proposed approach. We showed a QD s-shell resonance with a RF-signal to background ratio of at least 2.6 at QD saturation. Off-resonant mode-QD coupling, as well as cavity-mediated coupling between QDs, was observed. Regarding the latter, it was shown to be elemental that one of the interacting QDs is on resonance with the cavity mode. This opens up the possibility of preparation of an s-shell QD state by pumping resonantly the ground state of another QD (off-resonant with respect to the cavity mode) providing efficient off-resonant excitation (not achievable through the FM due to orthogonal orientation of the laser field). Additionally, we showed that the QD s-shell saturates at a QD specific resonant excitation density, at which also the RF-signal to background ratio is optimal.

An interesting opportunity enabled by the sample design are experiments in which the coupling of single and multiple QDs to the FM, especially in the limit of strong coupling between QD and excitation laser field [35] as well as between QD and FM [5] are dominant. This kind of system with a single QD coupled simultaneously to two competing oscillators - namely the excitation laser field and the cavity mode - has not yet been studied experimentally or theoretically, and is a promising candidate for novel nonlinear light-matter interaction effects. Moreover, it would be interesting to explore the preparation of a resonant QD state via s-shell resonant pumping of an off-resonant QD. This could be especially interesting if performed in time-dependent excitation experiments, as it would possibly enable deeper insight into the dynamics of the different coupling mechanisms.

\section{Acknowledgments}

The authors acknowledge financial support by the German Research Foundation DFG via Projects No. Ka2318/4-1 and No. Re2974/3-1, the SFB 787 "Semiconductor Nanophotonics: Materials, Models, Devices", and the State of Bavaria. A.M. appreciates support from the Polish Ministry of Science and Higher Education within the "Mobilność Plus" program.

\section{References}

[1] Ed Jahnke F 2012 Quantum Optics with Semiconductor Nanostructures (Cambridge: Elsevier)

[2] Kavokin A V, Baumberg J J, Malpuech G and Laussy F P 2007 Microcavities (Oxford: Oxford University Press)

[3] Rakher M T 2008 Quantum Optics with Quantum Dots in Microcavities (Ann Arbor, MI: ProQuest)

[4] Vahala K J 2003 Optical microcavities Nature 424 839-846 
[5] Reithmaier J P, Sęk G, Löffler A, Hofmann C, Kuhn S, Reitzenstein S, Keldysh L V, Kulakovskii V D, Reinecke T L and Forchel A 2004 Strong coupling in a single quantum dotsemiconductor microcavity system Nature $\mathbf{4 3 2}$ 197-200

[6] Michler P, Kiraz A, Becher C, Schoenfeld W V, Petroff P M, Zhang L, Hu E and İmamoğlu A 2000 A Quantum Dot Single-Photon Turnstile Device Science 290 2282-5

[7] Farrow T, See P, Bennett A J, Ward M B, Atkinson P, Cooper K, Ellis D J P, Unitt D C, Ritchie D A and Shields A J 2008 Single-photon emitting diode based on a quantum dot in a micropillar Nanotechnology 19345401

[8] Ladd T D, Loock P, Nemoto K, Munro W J and Yamamoto Y 2006 Hybrid quantum repeater based on dispersive CQED interactions between matter qubits and bright coherent light New Journal of Physics 8184

[9] İmamoğlu A, Awschalom D D, Burkard G, DiVincenzo D P, Loss D, Sherwin M and Small A 1999 Quantum Information Processing Using Quantum Dot Spins and Cavity QED Physical Review Letters 83 4204-7

[10] Gérard J M, Barrier D, Marzin J Y, Kuszelewicz R, Manin L, Costard E, Thierry-Mieg V and Rivera T 1996 Quantum boxes as active probes for photonic microstructures: The pillar microcavity case Applied Physics Letters 69 449-51

[11] Heindel T, Schneider C, Lermer M, Kwon S H, Braun T, Reitzenstein S, Höfling S, Kamp M and Forchel A 2010 Electrically driven quantum dot-micropillar single photon source with $34 \%$ overall efficiency Applied Physics Letters 96011107

[12] Munnelly P, Heindel, T, Karow M M, Hofling S, Kamp M, Schneider C and Reitzenstein S 2015 A Pulsed Nonclassical Light Source Driven by an Integrated Electrically Triggered Quantum Dot Microlaser IEEE Journal of Selected Topics in Quantum Electronics 21 681-9

[13] Albert F, Hopfmann C, Reitzenstein S, Schneider C, Hofling S, Worschech L, Kamp M, Kinzel W, Forchel A and Kanter I 2011 Observing chaos for quantum-dot microlasers with external feedback Nature Communications 2366

[14] Ates S, Ulrich S M, Ulhaq A, Reitzenstein S, Löffler A, Höfling S, Forchel A and Michler P 2009 Non-resonant dot-cavity coupling and its potential for resonant single-quantum-dot spectroscopy Nature Photonics 3 724-8

[15] Ding X, He Y, Duan Z C, Gregersen N, Chen M C, Unsleber, S, Maier S, Schneider C, Kamp M, Höfling S, Lu C Y and Pan J W 2016 On-Demand Single Photons with High Extraction Efficiency and Near-Unity Indistinguishability from a Resonantly Driven Quantum Dot in a Micropillar Physical Review Letters 116020401

[16] Unsleber S, Schneider C, Maier S, He Y M, Gerhardt S, Lu C Y, Pan J W, Kamp M and Höfling S 2015 Deterministic generation of bright single resonance fluorescence photons from a Purcellenhanced quantum dot-micropillar system Optics Express 23 32977-85

[17] Ates S, Ulrich S M, Reitzenstein S, Löffler A, Forchel A and Michler P 2009 Post-Selected Indistinguishable Photons from the Resonance Fluorescence of a Single Quantum Dot in a Microcavity Physical Review Letters 103167402

[18] Ed Michler P 2009 Single Semiconductor Quantum Dots (Berlin: Springer)

[19] Matthiesen C, Vamivakas A M and Atatüre M 2012 Subnatural linewidth single photons from a quantum dot Physical Review Letters 108093602

[20] Unsleber S, McCutcheon D P, Dambach M, Lermer M, Gregersen N, Höfling S, Mørk J, Schneider C and Kamp M 2015 Two-photon interference from a quantum dot microcavity: Persistent pure dephasing and suppression of time jitter Physical Review B 91075413

[21] Vamivakas A N, Lu C Y, Matthiesen C, Zhao Y, Fält S, Badolato A and Atatüre M 2010 Observation of spin-dependent quantum jumps via quantum dot resonance fluorescence Nature 467 297-300

[22] Müller M, Bounouar S, Jöns K D, Glässl M and Michler P 2014 On-demand generation of indistinguishable polarization-entangled photon pairs Nature Photonics 8 224-8

[23] Kuhlmann A V, Houel J, Brunner D, Ludwig A, Reuter D, Wieck A D and Warburton R J 2013 A dark-field microscope for background-free detection of resonance fluorescence from single semiconductor quantum dots operating in a set-and-forget mode Review of Scientific Instruments 84073905 
[24] Greuter L, Starosielec S, Najer D, Ludwig A, Duempelmann L, Rohner D and Warburton R J 2014 A small mode volume tunable microcavity: Development and characterization Applied Physics Letters 105121105

[25] Proux R, Maragkou M, Baudin E, Voisin C, Roussignol P and Diederichs C 2015 Measuring the Photon Coalescence Time Window in the Continuous-Wave Regime for Resonantly Driven Semiconductor Quantum Dots Physical Review Letters 114067401

[26] Reitzenstein S, Gregersen N, Kistner C, Strauss M, Schneider C, Pan L, Nielsen T R, Höfling S, Mørk J and Forchel A 2009 Oscillatory variations in the Q factors of high quality micropillar cavities Applied Physics Letters 94061108

[27] Schneider C, Gold P, Reitzenstein S, Höfling S and Kamp M 2016 Quantum dot micropillar cavities with quality factors exceeding 250,000 Applied Physics B 122 1-6

[28] Musiał A, Hopfmann C, Heindel T, Gies C, Florian M, Leymann H A M, Foerster A, Schneider C, Jahnke F, Höfling S, Kamp M and Reitzenstein S 2015 Correlations between axial and lateral emission of coupled quantum dot-micropillar cavities Physical Review B 91205310

[29] Nguyen H S, Sallen G, Voisin C, Roussignol Ph, Diederichs C and Cassabois G 2012 Optically gated resonant emission of single quantum dots Physical Review Letters 108057401

[30] Marzin J Y, Gérard J M, Izraël A, Barrier D and Bastard G 1994 Photoluminescence of single InAs quantum dots obtained by self-organized growth on GaAs Physical Review Letters 73716

[31] Purcell E M 1946 Spontaneous emission probabilities at radio frequencies Proceedings of the American Physical Society 69681

[32] Majumdar A, Kim E D, Gong Y, Bajcsy M and Vučković J 2011 Phonon mediated off-resonant quantum dot-cavity coupling under resonant excitation of the quantum dot Physical Review $B$ 84085309

[33] Kaniber M, Laucht A, Neumann A, Villas-Bôas J M, Bichler M, Amann M-C and Finley J J 2008 Investigation of the nonresonant dot-cavity coupling in two-dimensional photonic crystal nanocavities Physical Review B 77 161303(R)

[34] Winger M, Volz T, Tarel G, Portolan S, Badolato A, Hennessy K J, Hu E L, Bevertos A, Finley J, Savona V and Imamoğlu A 2009 Physical Review Letters 103207403

[35] Ulhaq A, Weller S, Ulrich S M, Roßbach R, Jetter M and Michler P 2012 Cascaded singlephoton emission from the Mollow triplet sidebands of a quantum dot Nature Photonics 6238 42 